\title{
Papers
}

\section{Prediction of survival for preterm births by weight and gestational age: restrospective population based study}

Elizabeth S Draper, Bradley Manktelow, David J Field, David James

\begin{abstract}
Objective To produce current data on survival of preterm infants.

Design Retrospective population based study. Setting Trent health region.

Subjects All European and Asian live births, stillbirths, and late fetal losses from 22 to 32 weeks' gestation, excluding those with major congenital malformations, in women resident in the Trent health region between 1 January 1994 and 31 December 1997.

Main outcome measures Birth weight and gestational age specific survival for both European and Asian infants $(a)$ known to be alive at the onset of labour, and (b) admitted for neonatal care.

Results 738 deaths occurred in 3760 infants born between 22 and 32 weeks' gestation during the study period, giving an overall survival rate of $80.4 \%$. The survival rate for the 3489 (92.8\%) infants admitted for neonatal care was $86.6 \%$. For European infants known to be alive at the onset of labour, significant variations in gestation specific survival by birth weight emerged from 24 weeks' gestation: survival ranged from 9\% (95\% confidence interval 7\% to 13\%) for infants of birth weight $250-499 \mathrm{~g}$ to $21 \%$ (16\% to $28 \%$ ) for those of 1000-1249 g. At 27 weeks' gestation, survival ranged from $55 \%$ (49\% to $61 \%$ ) for infants of birth weight $500-749 \mathrm{~g}$ (below the 10th centile) to $80 \%$ (76\% to $85 \%$ ) for those of $1250-1499 \mathrm{~g}$. Infants who were large for dates ( $\geqslant 27$ weeks' gestation) had a slightly reduced, but not significant, predicted survival. Similar survival rates were observed for Asian infants. The odds ratio for the survival of infants from a multiple birth compared with singleton infants was 1.4 (1.1 to 1.8). Survival graphs for infants admitted for neonatal care are presented by sex.

Conclusion Easy to use birth weight and gestational age specific predicted survival graphs for preterm infants facilitate decision making for clinicians and parents. It is important that these graphs are representative, are produced for a geographically defined population, and are not biased towards the outcomes of particular centres. Such graphs, produced in two stages, allow for the changing pattern of survival of infants from the start of the intrapartum period to immediately after admission for neonatal care.
\end{abstract}

\section{Introduction}

Data on the probability of survival of infants in high risk pregnancies can be of great value in guiding management. This information can help both clinical staff and parents to decide if and when to intervene in a pregnancy. Unit based data on the survival of preterm infants by birth weight and gestation can be easily compiled, but such data are easily biased by variation in local casemix and local variations in attitude to the care of the most immature infants. ${ }^{1}$ To overcome these problems, data on birth weight and gestational age specific survival should be derived from geographically defined populations using data from all pregnancies within the relevant gestation band.

Geographically based graphs for liveborn infants were produced in the 1980s for a US population ${ }^{2}$ and for the Netherlands, ${ }^{3}$ but both are now out of date as they were produced before the introduction of many important advances in perinatal care such as antenatal steroids and exogenous surfactant therapy. Birth weight and gestational age specific survival graphs have yet to be produced for a UK population. Attempts to produce such graphs have been hampered by the absence of gestational age in the statutory dataset required by the Office of National Statistics.

We aimed to produce birth weight and gestational age specific survival graphs for a population of infants born between 22 and 32 weeks' gestation in the United Kingdom. To maximise clinical relevance, we focused on two specific clinical situations. We aimed (a) to develop one set of data to indicate the chance of an infant being discharged alive from the neonatal service if it was known to be alive at either the onset of labour or when the decision to deliver was made, and $(b)$ to develop a second set of data to describe the sex specific survival to discharge home of infants admitted to a neonatal unit.

\section{Subjects and methods}

\section{Subjects}

We studied Trent health region, a geographically defined population, which comprises around 4.6 million people and about 60000 births a year. We included all births and late fetal losses from 22 to 32 weeks' gestation inclusive to mothers resident in the Trent health region between 1 January 1994 and 31 December 1997. We excluded infants of less than 22

\author{
Department of \\ Epidemiology and \\ Public Health, \\ Leicester University \\ Medical School, \\ Leicester LE1 6TP \\ Elizabeth S Draper \\ senior research fellow \\ in perinatal \\ epidemiology \\ Bradley Manktelow \\ medical statistician \\ Department of \\ Child Health, \\ Leicester Royal \\ Infirmary, Leicester \\ LE2 7LX \\ David J Field \\ professor of neonatal \\ medicine \\ Department of \\ Obstetrics and \\ Gynaecology, \\ Queens Medical \\ Centre, University \\ of Nottingham, \\ Nottingham \\ NG7 2UH \\ David James \\ professor of \\ fetomaternal medicine \\ Correspondence to: \\ E S Drapermsn@ \\ le.ac.uk
}

BMJ 1999;319:1093-7 
weeks' gestation as there were no survivors in this group. The Trent confidential inquiry into stillbirths and deaths in infancy (part of the national confidential inquiry into stillbirths and deaths in infancy programme $^{4}$ ) provided data on all late fetal losses of 22 and 23 weeks' gestation, all stillborn infants known to be alive at the onset of labour, and all infant deaths before discharge from neonatal care, and the Trent neonatal survey ${ }^{5}$ provided data for all infants of $\leqslant 32$ weeks' gestation admitted to the 16 neonatal intensive care units in the Trent health region.

We collected data on date of birth, birth weight, gestation, postcode, birth status (live born or stillborn), and survival status of the infant at discharge from the labour ward or neonatal unit, and we collected details of other characteristics that might influence survivalsex, ethnic origin, and whether the infant was from a multiple pregnancy. We defined gestation according to the hierarchy specified by the national confidential inquiry into stillbirths and deaths in infancy programme: mother certain of her dates (most reliable); early dating scan (less than 20 weeks' gestation); late dating scan (more than 20 weeks' gestation); and postnatal examination (least reliable). If the difference between maternal date and early dating scan was more than 7 days, we chose the early dating scan. The two datasets were merged and validated. To ensure that infants were only counted once, we deleted any duplicate records (infants admitted to a neonatal unit who subsequently died were represented in both datasets). Infants with lethal congenital anomalies were excluded from the analysis.

\section{Statistical analysis}

We analysed data for two time periods: all infants known to be alive at the onset of labour or when the decision to deliver was made; and a subset of this group of all infants admitted to neonatal units. As studies have shown that ethnic differences have a major influence on outcome, ${ }^{6-8}$ we calculated the birth weight and gestational age specific survival for both Asian (originating from the Indian subcontinent) and European infants within each

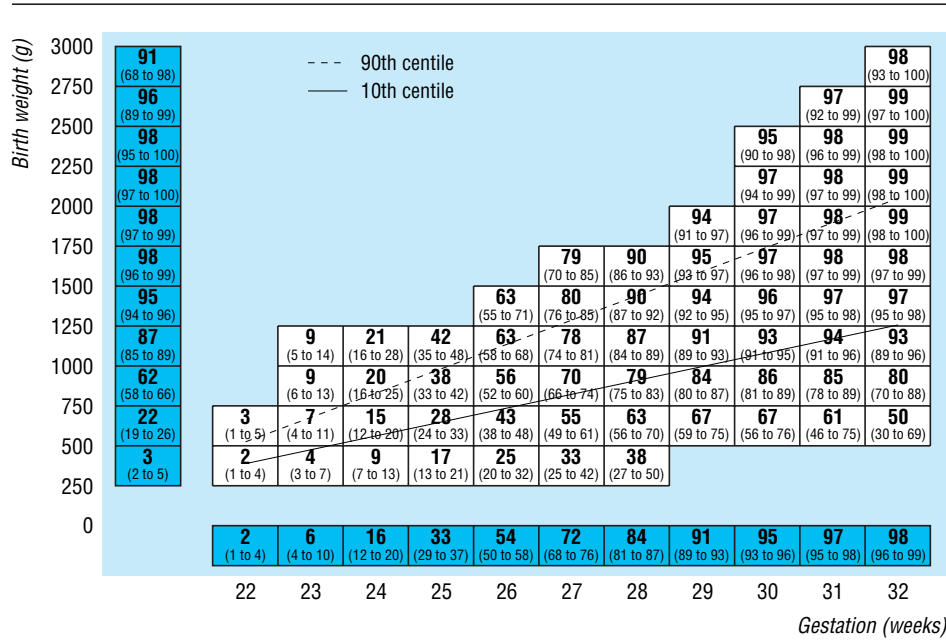

Fig 1 Median (95\% confidence interval) predicted percentage survival for European infants known to be alive at onset of labour. Values above 90th centile represent infants large for gestational age, values below 10th centile represent infants small for gestational age group. We excluded infants from other ethnic groups owing to small numbers.

We calculated the mean birth weight for gestation for all infants, then included ethnic group as a two level factor in a weighted linear regression model of birth weight and gestation. The difference between the observed and expected birth weight of each infant was then calculated and used within a logistic regression model of survival. Multiplicity was included in the model as a binary variable (singleton $v$ multiple) to calculate the odds ratio for survival in infants from multiple compared with singleton pregnancies-we then used this to develop a simple equation for use in clinical practice. The fit of the logistic model was assessed with the Hosmer and Lemeshow goodness of fit test, ${ }^{9}$ with $\mathrm{P}<0.05$ taken to be evidence of a statistically significant difference between observed and predicted survival. We then constructed a graph showing the predicted proportional survival (with 95\% confidence intervals) of singleton infants by birth weight increments of $250 \mathrm{~g}$ and gestational age intervals of 1 week both separately and together. Graphs for infants admitted to neonatal care were constructed by sex for the European but not Asian population as the sample size of the latter was too small. Distribution free smoothed lines were added to the graphs to show the observed 10th and 90th centiles for birth weight.

\section{Results}

\section{Study population}

Between 1 January 1994 and 31 December 1997 there were over 240000 live births, stillbirths, and late fetal losses of $\geqslant 22$ weeks' gestation within Trent health region. Of these, 3871 infants were $\leqslant 32$ weeks' gestation and known to be alive at the onset of labour. We excluded six infants with missing data for birth weight, and 115 infants with lethal congenital anomalies, leaving 3760 infants of which 944 (25.1\%) were from multiple pregnancies. Overall, there were 738 deaths giving an overall survival rate of $80.4 \%$, ranging from $6 \%$ for infants of 23 weeks' gestation to $98 \%$ for those of 32 weeks' gestation. In total, 271 infants were either intrapartum stillbirths or died shortly after delivery leaving 3489 (92.8\%) infants admitted to neonatal units: $3162(86.6 \%)$ of these infants survived to discharge home from neonatal care.

Around $36 \%$ of gestations were corrected after a dating ultrasound scan at $<20$ weeks' gestation. No terminal digit preference for even numbers in gestational age was noted.

\section{European infants}

Figure 1 shows the proportional survival of singleton European infants known to be alive at the onset of labour. The Hosmer and Lemeshow goodness of fit statistic $^{9}$ for this model was $\chi^{2}=7.06, \mathrm{df}=8, \mathrm{P}=0.53$. At 22 weeks' gestation predicted survival of European infants was 2\%-3\% irrespective of their size. Significant variations in gestation specific survival by birth weight emerged from 24 weeks' gestation: predicted survival ranged from $9 \%$ (7\% to $13 \%$ ) for birth weights of 250 $499 \mathrm{~g}$ to $21 \%$ (16\% to $28 \%$ ) for those of $1000-1249 \mathrm{~g}$. At 28 weeks' gestation predicted survival was $63 \%$ (56\% to $70 \%$ ) for birth weights of 500-749 $\mathrm{g}$ (below the 10th centile) and $90 \%(87 \%$ to $92 \%)$ for those of 1250 - 
1499 g. At 32 weeks' gestation predicted survival was $80 \%$ (70\% to $88 \%)$ for birth weights of $750-999 \mathrm{~g}$ and $98 \%$ (97\% to 99\%) for those of $1500-2499 \mathrm{~g}$. A reduced predicted survival at gestations of $\geqslant 27$ weeks was seen in infants large for dates, although this finding was not significant.

\section{Asian infants}

Figure 2 shows the predicted survival of Asian infants known to be alive at the onset of labour, derived from the same model as figure 1 . As with the European infants, only 2\%-3\% of Asian infants of 22 weeks' gestation were predicted to survive irrespective of their size. Predicted survival at 28 weeks' gestation was $69 \%$ (63\% to 74\%) for birth weights of 500-749 $\mathrm{g}$ (below the 10th centile) and $90 \%(87 \%$ to $92 \%)$ for those of 1250 1499 g. Predicted survival at 32 weeks' gestation was $96 \%$ (93\% to $97 \%$ ) for birth weights of $750-999 \mathrm{~g}$ and $99 \%(98 \%$ to $100 \%)$ for those of $1500-2499 \mathrm{~g}$.

\section{Infants from multiple births}

Infants from multiple births had, for the same birth weight, gestation, and ethnic origin as singleton infants, a greater chance of survival (odds ratio 1.4, 1.1 to 1.8). From this odds ratio we developed a simple equation for use in clinical practice to adjust for the predicted survival of multiple births (to the nearest percentage point):

$$
P_{\mathrm{m}}=\frac{375 \times P_{\mathrm{s}}}{275+P_{\mathrm{s}}}
$$

where $P_{\mathrm{s}}$ is the predicted percentage survival for singletons and $P_{\mathrm{m}}$ is the predicted percentage survival for infants of multiple birth. This formula was also found to apply to infants admitted for neonatal care (1.35, 1.0 to 1.8$)$.

Figures 3 and 4 show the predicted survival of male and female European infants admitted for neonatal care. Female infants had an overall better predicted survival $(0.7,0.5$ to 0.9$)$ - for example, at 24 weeks' gestation the predicted survival of male infants was $22 \%$ (17\% to 27\%) for birth weights of 500-749 $\mathrm{g}$ and 29\% (24\% to $35 \%$ ) for those of $750-999 \mathrm{~g}$ whereas the predicted survival of female infants was 29\% (23\% to $35 \%$ ) and $37 \%$ (30\% to $44 \%)$ respectively. Owing to the small number of Asian infants $(n=232)$, figure 5 shows the predicted survival for all Asian infants (not subdivided by sex) admitted for neonatal care.

\section{Discussion}

Data on gestational age is not collected routinely in the United Kingdom. Consequently it has been difficult to provide national data on survival after preterm delivery, despite the need for such information when counselling women with an anticipated preterm delivery. The improving prognosis for infants of very short gestations makes it important to give parents an accurate prediction of outcome for their child. ${ }^{10}$ Our data provide gestational age specific survival rates for singleton Asian and European infants, by birth weight groupings, for a geographically defined population, at two separate time periods-all infants known to be alive at the onset of labour, when the sex of the infant is generally unknown; and a subset of this group of all infants admitted to neonatal units. Trent health region

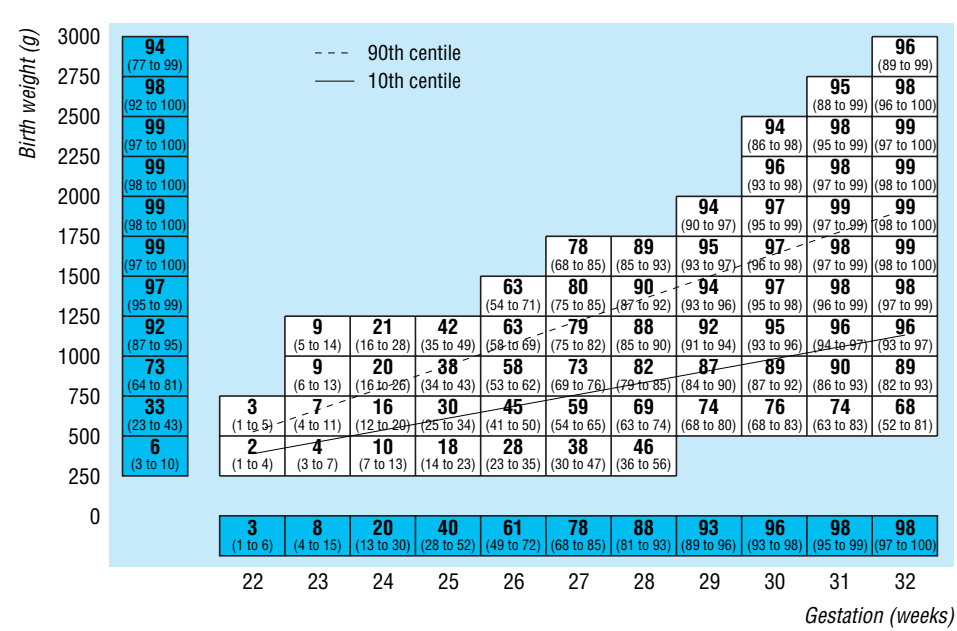

Fig 2 Median (95\% confidence interval) predicted percentage survival for Asian infants known to be alive at the onset of labour. Values above 90th centile represent infants large for gestational age, values below 10th centile represent infants small for gestational age

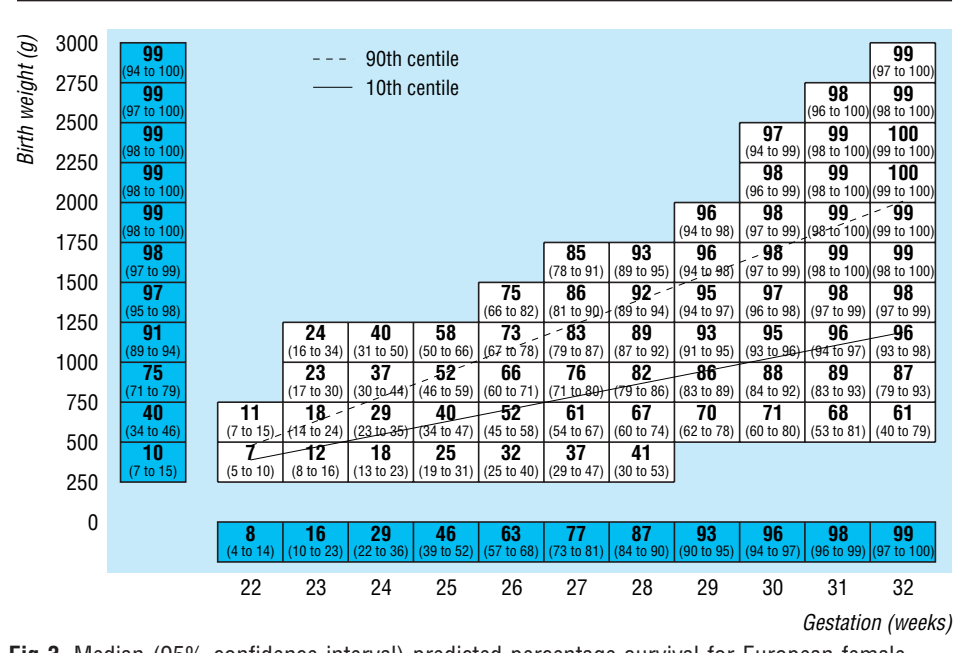

Fig 3 Median (95\% confidence interval) predicted percentage survival for European female infants admitted for neonatal care. Values above 90th centile represent infants large for gestational age, values below 10th centile represent infants small for gestational age

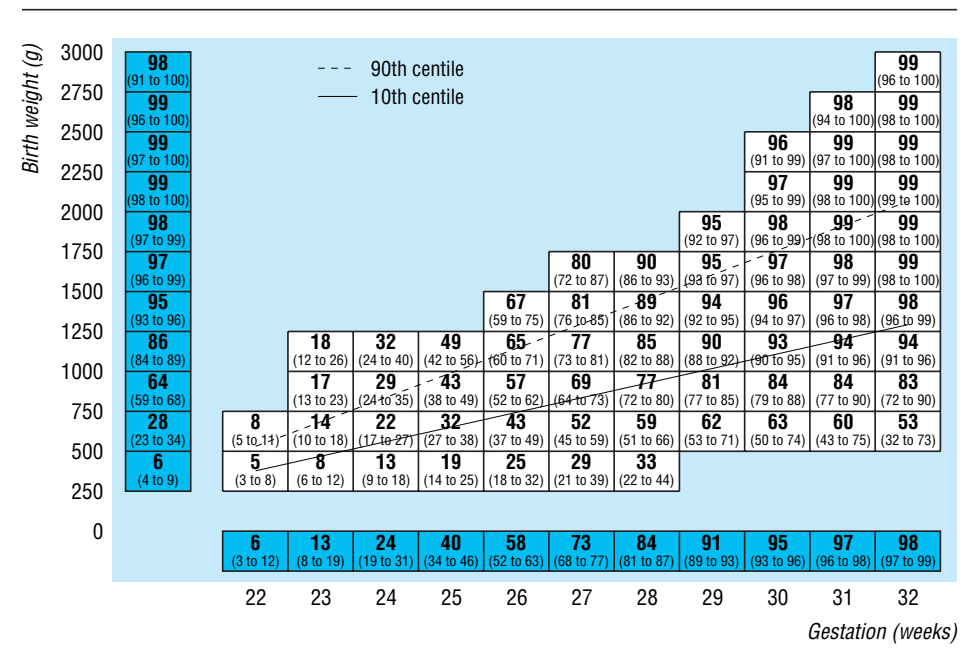

Fig 4 Median (95\% confidence interval) predicted percentage survival for European male infants admitted for neonatal care. Values above 90th centile represent infants large for gestational age, values below 10th centile represent infants small for gestational age 


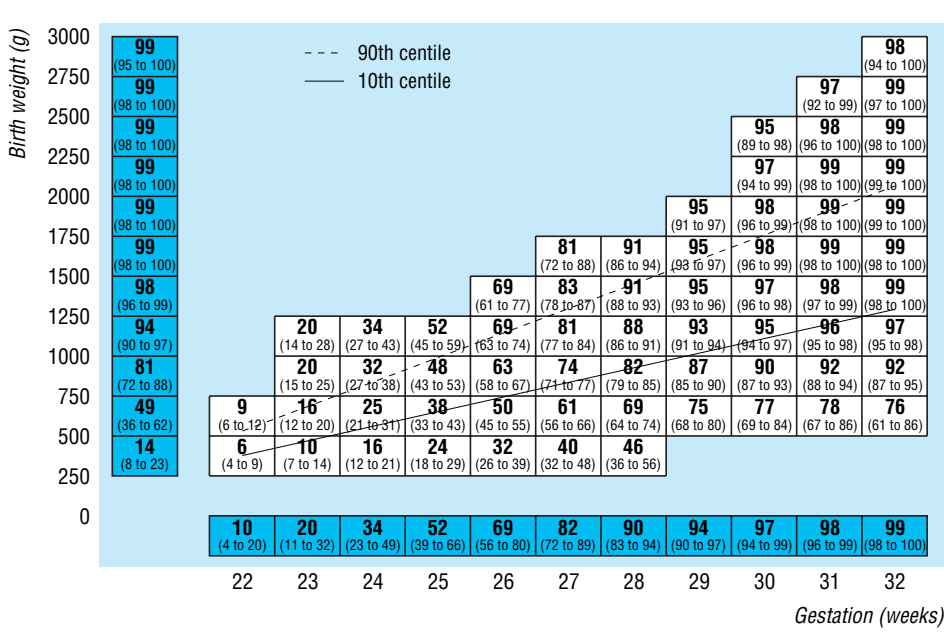

Fig 5 Median (95\% confidence interval) predicted percentage survival for Asian infants admitted for neonatal care. Values above 90th centile represent infants large for gestational age, values below 10th centile represent infants small for gestational age is considered representative of England and Wales, with a mixture of urban and rural populations and similar mortality and morbidity measures-for example, in 1996 the perinatal mortality rate was 8.6 per 1000 births for England and Wales and 8.7 per 1000 births for Trent, and the proportion of babies weighing less than $2500 \mathrm{~g}$ was $7.3 \%$ for both England and Wales and Trent.

\section{Additional factors affecting infant survival}

Factors known to affect the survival of infants (ethnic origin, sex, and multiple pregnancy) were included in the modelling process. For ease of use, separate graphs were produced for European and Asian infants. Previous reports indicating improved survival of female infants requiring intensive care are confirmed in our study among European infants. ${ }^{112}$

We produced simple equations for each set of graphs, adjusting for the predicted survival of multiple births. The finding of a better outcome for multiple births was unexpected and is the reverse of the situation in mature infants, although a finding of no difference in the survival between preterm singletons and twins has been suggested, controlled for birth weight and gestational age at delivery. ${ }^{13}$ It would seem that other causes of preterm delivery, such as growth restriction, placental abruption, and infection, may be associated with negative effects on viability that are significant when such preterm infants are compared with those precipitated by multiple pregnancy, where multiple birth itself is often the most important factor that leads to preterm birth. We could identify no clinically or biologically plausible theory that might lead to such pregnancies being systematically wrongly assessed for maturity or other factors that might significantly affect survival.

\section{International perspectives}

Several other studies have looked at birth weight and gestational age specific survival, all of which have limitations. In two US studies ${ }^{214}$ geographically based survival graphs were produced for liveborn infants. The first study, ${ }^{2}$ however, was carried out before the introduction of many important new management strategies-for example, exogenous surfactant therapy and the widespread use of antenatal steroids. In the second study, ${ }^{14}$ the survival of infants was described by birth weight and gestation separately but not together. That study produced data for the actuarial survival of infants by weeks of gestation or $100 \mathrm{~g}$ birth weight groupings and showed that the survival in the smallest infants improved dramatically during the first few days of life although the risk of a late death was high in the smallest of these infants. As a result we are planning to supplement our current data with a third set of graphs including late deaths among those infants surviving the perinatal period, as well as investigating the prediction of morbidity in preterm infants. A further geographically based study reported for the national livebirth cohort of the Netherlands in $1983^{3}$ is also now of limited relevance. Geographically defined studies ${ }^{15} 16$ of the survival of preterm infants in the United Kingdom have either concentrated on gestation specific or birth weight specific survival alone or have considered the total population of deliveries including deaths before the onset of labour. None of these studies present data for all infants known to be alive at the onset of labour and therefore are of limited use to obstetricians.

The importance of producing birth weight and gestational age specific data is highlighted by the variation in birth weight specific mortality by week of gestation, apparent from as early as 24 weeks' gestation in each of the graphs. This allows for a more accurate prediction of the survival of infants depending on whether they are small, appropriate, or large for dates.

\section{Conclusion}

Birth weight and gestational age specific predicted survival graphs for preterm infants facilitate decision making for obstetricians, neonatologists, and parents and give a range of predicted survival for any given gestational age by estimated or actual birth weight. It is important that such graphs are produced for a geographically defined population so that they are

\section{Key messages}

- Birth weight and gestational age specific predicted survival graphs for preterm infants facilitate decision making for clinicians and parents

- Survival graphs should be representative and not biased towards the outcomes of particular centres

- Period specific graphs allow for the changing pattern of survival from the start of the intrapartum period to the immediate period after admission for neonatal care

- Causes of preterm delivery in singletons may lead to a poorer survival rate (controlled for gestation and birth weight) than those precipitated by multiple pregnancy

- Survival graphs need regular updating to allow for improvements in survival of infants 
representative and not biased towards the outcomes of particular centres. The production of such graphs in two stages allows for the changing pattern of survival from the start of the intrapartum period to the immediate period after admission for neonatal care. A continual process of updating needs to be in place to allow for improvements in survival of infants.

We thank Sue Wood (regional coordinator), Jayne Bennett (administrator), and all the district coordinators for the Trent confidential inquiry into stillbirths and deaths in infancy, the staff (medical, nursing, and clerical) of the 16 perinatal units in Trent, those units adjacent to Trent that allowed us access to data on cross boundary flows of patients, and Keith Abrams and Nicky Spiers for their statistical advice.

Contributors: DJ, ESD, and DJF conceived the original idea for the study; they will act as guarantors for the paper. All authors contributed to the writing of the paper. BM carried out the statistical analysis.

Funding: The Trent neonatal survey and Trent confidential inquiry into stillbirths and deaths in infancy are funded through the Trent regional office

Competing interests: None declared.

1 Field DJ, Hodges S, Mason E, Burton P, Yates J, Wale S. The demand for neonatal intensive care. $B M J$ 1989;299:1305-8.

2 Copper RL, Goldenberg RL, Creasy RK, DuBard MB, Davis RO, Entman $\mathrm{SS}$, et al. A multicenter study of preterm birth weight and gestational agespecific neonatal mortality. Am J Obstet Gynecol 1993;168:78-84

3 Verloove-Vanhorick SP, Verwey RA, Brand R, Gravenhorst JB, Keirse
MJNC, Ruys JH. Neonatal mortality risk in relation to gestational age and birthweight. Lancet 1986;1:55-7.

4 Clarke M, Draper ES, James D, McKeever P, Perkins MJ, Wood S. Confidential enquiry into stillbirths and deaths in infancy (CESDI) 1994-5-one of the Trent infant mortality and morbidity studies. Leicester: Trent Institute for Health Services Research; 1996.

5 Burton P, Draper ES, Fenton A, Field DJ. Neonatal intensive care cots: estimating the population-based requirement in Trent UK. $J$ Epidemiol Community Health 1995; 48:617-28.

6 Greenberg DN, Yoder BA, Clark RH, Butzin CA, Null DM. Effect of maternal race on outcome of preterm infants in the military. Pediatric. 1993;91:572-7.

7 Yuksel B, Greenough A. Ethnic origin and lung function in infants born prematurely. Thorax 1995;50:773-6.

8 Fuentes-Afflick E, Hessol NA. Impact of Asian ethnicity and national origin on infant birthweight. Am J Epidemiol 1997;145:148-55.

9 Hosmer DW, Lemeshow S. Goodness of fit tests for the multiple logistic regression model. Commun Statist Theor Methods 1980;A9:1043-69.

10 Rennie JM. Perinatal management at the lower margin of viability. Arch Dis Child 1996;74:214-8F

11 Field D, Milner AD, Hopkin IE, Madeley RJ. Changing overall workload in neonatal units. $B M J 1985 ; 290: 1539-42$

12 Field D, Milner AD, Hopkin IE, Madeley RJ. The changing pattern of neonatal respiratory disease. Pediatr Pulmomol 1987;8:231-5.

13 Wolf EJ, Vintzileos AM, Rosenkranz TS, Rodis JF, Lettieri L, Mallozzi A. A comparison of pre-discharge survival and morbidity in singleton and twin very low birth weight infants. Obsterics Gynecol 1992;80:436-9.

14 Cooper TR, Berseth CL, Adams JM, Weisman LE. Acturial survival in the premature infant less than 30 weeks' gestation. Pediatrics 1998;101:975-8.

15 Tin W, Wariyar U, Hey E for the northern neonatal network. Changing prognosis for babies of less than 28 weeks' gestation in the north of England between 1983 and 1994. BMJ 1997;314:107-11.

16 Cartlidge PH, Stewart JH. Survival of very low birthweight and very preterm infants in a geographically defined population. Acta Paediat 1997;86:105-10.

(Accepted 13 July 1999)

\section{Two view mammography at incident screens: cost effectiveness analysis of policy options}

Katharine Johnston, Jackie Brown

\begin{abstract}
Objective To determine the cost effectiveness of two view mammography at incident screens.

Design Incremental cost effectiveness analyses recognising differences in current reading policy, based on effectiveness data from an observational study.

Setting Breast screening programmes in England and Wales.

Main outcome measures Health service costs, cancers detected, incremental cost effectiveness ratios per cancer detected, whole time equivalent staff. Results For programmes currently using one view with some form of double reading, the incremental cost effectiveness ratio of two view mammography at incident screens ranged between $£ 6589$ and $£ 6716$, depending on the reading policy. For programmes currently using one view with single reading, two policy options were found to be more efficient than two view single reading: one view with double reading (arbitration; incremental cost effectiveness ratio of $£ 210$ ) and two view double reading (arbitration). If programmes using one view with single reading changed to double reading (arbitration) and then subsequently to two views double reading (arbitration), additional cancers could be detected with an incremental cost effectiveness ratio of $£ 7983$. The implementation cost of two view mammography at
\end{abstract}

incident screens in programmes in England and Wales would be £2.9 million and would require 13.4 whole time equivalent radiologists.

Conclusions The cost effectiveness of two view mammography at incident screens depends on the film reading policy. A policy of two view mammography at incident screens in England and Wales would be efficient only if programmes using single reading moved to double reading. Given limited resources, priority should be given to introducing double reading in the subset of programmes currently using single reading as this requires fewer additional radiologists and is more cost effective.

\section{Introduction}

The NHS breast screening programme was introduced in 1987 after the recommendations of the Forrest report. ${ }^{1}$ The recommended policy was to invite women between the ages of 50 and 64 years for mammographic screening every three years with one view mammography. The report concluded that the age range, frequency of screening, and screening methods should be kept under review. It was also recommended that an economic component should be built into studies of these policy options as the costs and benefits of screening would be affected.
Health Economics Research Centre, University of Oxford, Institute of Health Sciences, Headington, Oxford OX3 7LF

Katharine Johnston research fellow

Health Economics Research Group, Brunel University, Uxbridge,

Middlesex

UB8 3PH

Jackie Brown

senior research fellow

Correspondence to: K Johnston kathy.johnston@his. ox.ac.uk

BMJ 1999;319:1097-102 